\title{
Effect of Scattering on the Transmission of Si Nanorod Arrays
}

\author{
Xiaojian Yu, Chaogang Lou \\ Joint International Research Laboratory of Information Display and Visualization, School of Electronic Science and Engineering, \\ Southeast University, Nanjing, China \\ Email:1cg@seu.edu.cn
}

How to cite this paper: Yu, X.J. and Lou, C.G. (2019) Effect of Scattering on the Transmission of Si Nanorod Arrays. Journal of Materials Science and Chemical Engineering, 7, 65-70. https://doi.org/10.4236/msce.2019.712008

Received: October 6, 2019

Accepted: December 17, 2019

Published: December 20, 2019

\begin{abstract}
The effect of scattering on the transmission of subwavelength Si nanorod arrays is investigated. It is found that the distance between the nanorods has important effect on the transmission because the scattered light varies largely with it. When the nanorods are close to each other, the back-scattered light and the light reflected by the surface of substrates interfere destructively and lead to a high transmission. When the distance of the nanorods increases, the intensity of the scattered light decrease and this makes the transmission decrease. The reflection and the transmission of the nanorod arrays can be adjusted by changing the distance between the neighbouring nanorods.
\end{abstract}

\section{Keywords}

Nanorod, Transmission, Interference, Back-Scattered

\section{Introduction}

In past decades, subwavelength nanorod arrays attracted much attention because they can lower largely the reflection of light [1] [2] [3] [4]. A lot of works have been done in this area, involving materials [5] [6] [7], structures [8] [9] [10] [11] and fabrication [12] [13] [14] [15], etc. In order to understand their light-trapping mechanism, many efforts have also been done to explain the anti-reflection of the nanorod arrays [16]-[21].

In this work, we investigate the effect of scattering of light on the transmission of the subwavelength nanorod arrays. By changing the distance between the neighbouring nanorods, the interference between the light scattered by the nanorods and the light reflected by the surface of substrates is adjusted. As a result, the transmission of the nanorod arrays varies with the distance between the neighbouring nanorods. 


\section{Theoretical Model}

Figure 1(a) and Figure 1(b) shows the schematic of the periodic nanorod arrays and the simulated model, respectively. In the simulation, Si nanorods are located on Si substrates. The radius of the nanorods is set as $50 \mathrm{~nm}$, and their height is $135 \mathrm{~nm}$. The top and bottom ends of the model are perfect matched layer (PML) to eliminate the influence of the reflection at the both ends. The other four faces have periodic boundary conditions. To avoid the effect of diffraction, incident wavelength is set as $1 \mu \mathrm{m}$, much longer than the period of the nanorod arrays. The real part of the refractive index of $\mathrm{Si}$ is 3.57 and its imaginary part is 0.001 . The direction of the incident light is perpendicular to the surface of the substrates.

\section{Results and Discussion}

Figure 2 gives the transmission of the nanorod arrays with the different distance between neighbouring nanorods. It can be seen that the transmission increases at first. After the distance between the neighbouring nanorods rises to $11 \mathrm{~nm}$, the transmission turns down. This may be explained by the interference between the light back-scattered by the nanorods and the light reflected by the surface of the substrates.
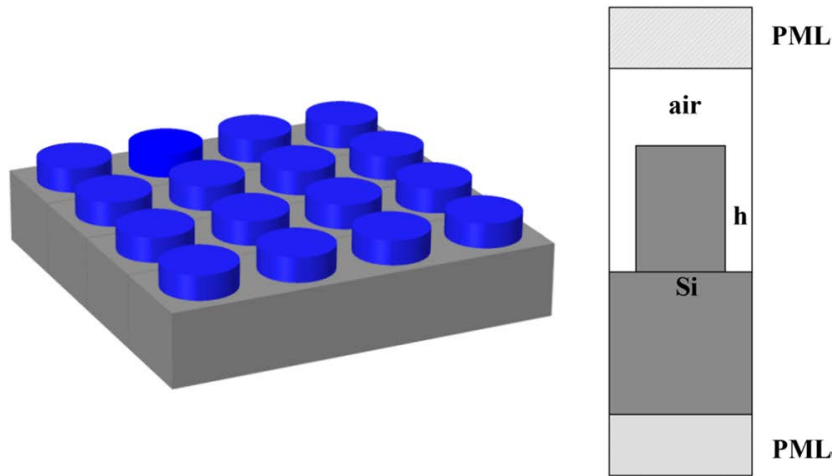

Figure 1. (a) Schematic of periodic Si nanorod arrays on Si substrates and (b) simulated model.

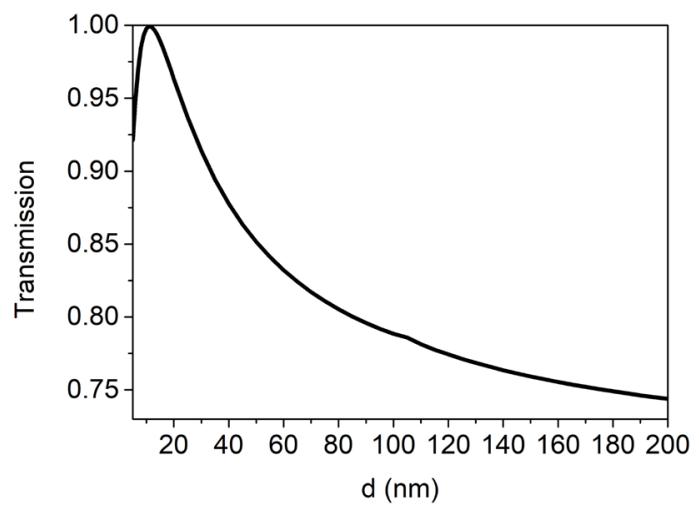

Figure 2. Transmission of the nanorod arrays with the different distance between neighbouring nanorods. 
Figure 3 gives the oscillograms of the reflected light and the back-scattered light of the nanorod arrays with different distance between the neighbouring nanorods. The abscissa denotes the distance from a point on the axis of the nanorod to the surface of the substrates. The chosen back-scattered light propagates along the axis of the nanorod. It can be seen that, the phase differences between the back-scattered light and the reflected light is greater than three quarters of $\pi$, so the interference between the back-scattered light and the reflected light is destructively. This indicates that the nanorod arrays can improve the transmission. Furthermore, the intensity of the back-scattered light decreases with the distance between the neighbouring nanorods, and this make the intensity of the resultant waves of the back-scattered light and reflected light increase, and leads to decreasing transmission.

When the distance between the neighbouring nanorods is $11 \mathrm{~nm}$, the intensity of the back-scattered light is nearly the same as that of the reflected light, and their phase difference is approximately equal to $\pi$. So they interfere destructively and the intensity of the resultant waves is nearly equal to zero. This is why the transmission at the distance of $11 \mathrm{~nm}$ reaches the maximum.

Figure 4 shows the pattern of the interference between the reflected light and

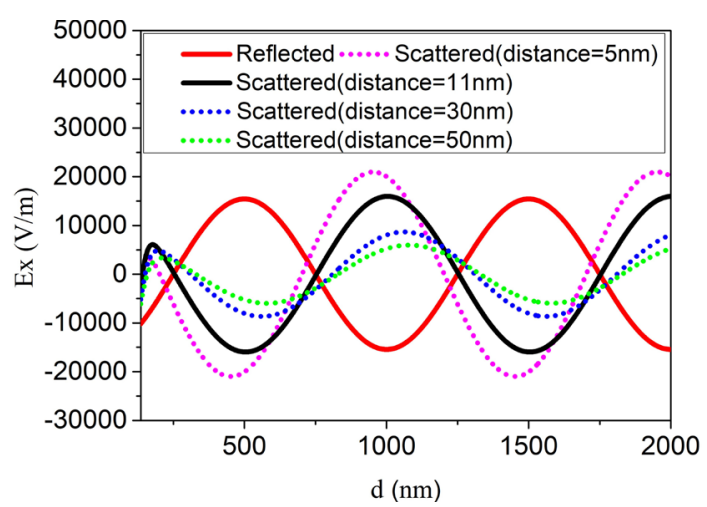

Figure 3. The oscillograms of the reflected light and the back-scattered light of the nanorod arrays with different distance between the neighbouring nanorods. The abscissa denotes the distance from a point on the axis of the nanorod to the surface of the substrates. The chosen back-scattered light propagates along the axis of the nanorod.

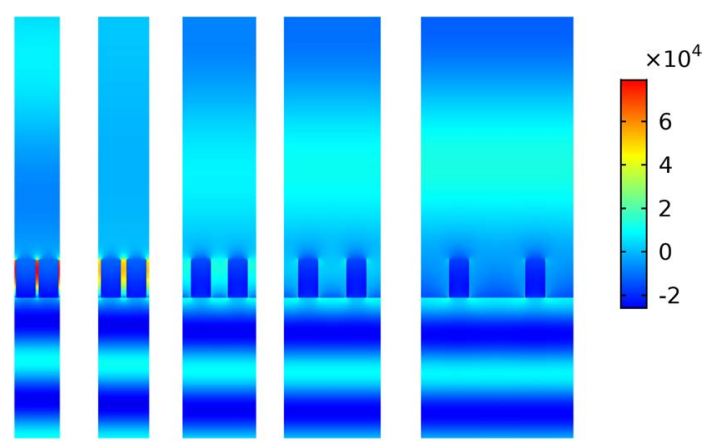

Figure 4. The pattern of the interference between the reflected light and the scattered light (the incident light is not included) of nanorod arrays with different distance between neighbouring nanorods. (a) $d=5 \mathrm{~nm}$, (b) $d=11 \mathrm{~nm}$, (c) $d=30 \mathrm{~nm}$, (d) $d=50 \mathrm{~nm}$, (e) $d=100 \mathrm{~nm}$. 
the scattered light (the incident light is not included because it does not influence the transmission) of the nanorod arrays with different distance between neighbouring nanorods. It can be seen that the different distance between the nanorods leads to the different interference. When the distance is $11 \mathrm{~nm}$, the resultant wave is weaker than others. This agrees with the results in Figure 3 where the intensity of the back-scattered light is similar to that of the reflected light and their phase difference is close to $\pi$.

It should be pointed that the above discussion is based on the fact that the scatter cross-section of the nanorods is smaller than the cross-section of the nanorods. If the scatter of the nanorods is strong enough to make the scatter cross-section become large and cover the whole surface of the substrates, the transmission will be influenced differently by the scatter. This is a complicated research work which we will focus on next.

\section{Conclusion}

The effect of scattering on the transmission of subwavelength Si nanorod arrays is demonstrated through the different distance between neighbouring nanorods. When the nanorods are close to each other, the back-scattered light and the light reflected by the surface of substrates interfere destructively and lead to a high transmission. When the distance of the nanorods increases, the intensity of the scattered light decreases, and this makes the transmission decrease. The reflection and the transmission of the nanorod arrays can be adjusted by changing the distance between the neighbouring nanorods.

\section{Acknowledgements}

The authors thank the supports from the Natural Science Foundation of Jiangsu (Grant No. BK2011033), the Primary Research \& Development Plan of Jiangsu Province (Grant No. BE2016175) and Chinese Postdoctoral Science Foundation (Grant No. 2017M621581).

\section{Conflicts of Interest}

The authors declare no conflicts of interest regarding the publication of this paper.

\section{References}

[1] Bernhard, C.G. and Miller, W.H. (1962) A Corneal Nipple Pattern in Insect Compound Eyes. Acta Physiologica Scan Dinavica, 56, 385-386. https://doi.org/10.1111/j.1748-1716.1962.tb02515.x

[2] Yoshidah, A., Motoyama, M., Kosaku, A. and Miyamoto, K. (1997) Antireflective Nano Protuberance Array in the Transparent Wing of the a Hawk Moth, Cephonodes Hylas. Zoological Science, 14, 737-741. https://doi.org/10.2108/zsj.14.737

[3] Kang, S.M., Jang, S., Lee, J.K., Yoon, J.J., Yoo, D.E., Lee, J.W., Choi, M. and Park, N.G. (2016) Moth-Eye $\mathrm{Tio}_{2}$ Layer for Improving Light Harverting Efficiency in Perovskite Solar Cells. Small, 12, 2443-2449. https://doi.org/10.1002/smll.201670094 
[4] Peer, A., Biswas, R., Park, J., Shinar, M.R. and Shinar, J. (2017) Light Management in Perovskite Solar Cells and Organic LEDs with Microlens Arrays. Optics Express, 25, 10704-10709. https://doi.org/10.1364/oe.25.010704

[5] Margueritat, J., Gonzalo, J.C., Afonso, N., Mlaysh, A., Murray, D.B. and Saviot, L. (2006) Surface Plasmons and Vibrations of Self-Assembled Silver Nanocolumns. Nano Letters, 6, 2037-2042. https://doi.org/10.1021/nl061237h

[6] Hernández-Jiménez, A., Hernández-Santiago, J. and Macias-GarcíA, A. (2002) Relaxation Modulus in PMMA and PTFE Fitting by Fractional Maxwell Model. Polymer Testing, 21, 325-331. https://doi.org/10.1016/s0142-9418(01)00092-7

[7] Brongersma, M.L., Cui, Y. and Fan, S.H. (2014) Light Management for Photovoltaics Using High-Index Nanostructures. Nature Materials, 13, 451-460. https://doi.org/10.1038/nmat3921

[8] Chang, C.H., Caballero, J.D., Choi, H.J. and Barbastathis, G. (2011) Nanostructured Gradient-Index Antireflection Diffractive Optics. Opt. Lett, 36, 2354-2356. https://doi.org/10.1364/ol.36.002354

[9] Song, Y.M., Yu, J.S. and Lee, Y.T. (2010) Antireflective Submicrometer Gratings on Thin-Film Silicon Solar Cells for Light-Absorption Enhancement. Opt. Lett, 35, 276-278. https://doi.org/10.1364/ol.35.000276

[10] Huang, Y. and Chattopadhyay, F.S. (2013) Nanostructure Surface Design for Broadband and Angle-Independent Antireflection. Journal of Nanophotonics, 7, 941-949. https://doi.org/10.1117/1.jnp.7.073594

[11] Yang, L.Y., Feng, Q., Ng, B.H., Luo, X.G. and Hong, M.H. (2010) Hybrid Moth-Eye Structures for Enhanced Broadband Antireflection Characteristics. Applied Physics Express, 3, 26021-26023. https://doi.org/10.1143/apex.3.102602

[12] Choi, K., Park, S.H., Song, Y.M., Lee, Y.T., Hwangbo, C.K., Yang, H. and Lee, H.S. (2010) Nano-Tailoring the Surface Structure for the Monolithic High-Performance Antireflection Polymer Film. Adv. Mater, 22, 3713-3718. https://doi.org/10.1002/adma.201001678

[13] Sun, C.H., Jiang, P. and Jiang, B. (2008) Broadband Moth-Eye Antireflection Coatings on Silicon. Appl. Phys. Lett, 92, 1112 1-3. https://doi.org/10.1063/1.2870080

[14] Min, W.L., Jiang, B. and Jiang, P. (2008) Bioinspired Self-Cleaning Antireflection Coatings. Adv. Mater, 20, 14-18. https://doi.org/10.1002/adma.200800791

[15] Lalanne, P. and Morris, G.M. (1997) Antireflection Behavior of Silicon Subwavelength Periodic Structures for Visible Light. Nanotechnology, 8, 53-56. https://doi.org/10.1088/0957-4484/8/2/002

[16] Jing, X.F., Shao, J.D., Jin, Y.X. and Fan, Z.X. (2012) Near-Field Distribution of Broadband Antireflective Nanostructure Arrays. Optik, 123, 527-533. https://doi.org/10.1016/j.ijleo.2011.05.019

[17] Giordano, S. (2003) Effective Medium Theory for Dispersions of Dielectric Ellipsoids. Journal of Electrostatics, 58, 59-76. https://doi.org/10.1016/s0304-3886(02)00199-7

[18] Bruggeman, D.A.G. (2006) Berechnung Verschiedener Physikalischer Konstanten von Heterogenen Substanzen. II. Dielektrizitätskonstanten und Leitfähigkeiten von Vielkristallen der Nichtregulären Systeme. Annalen Der Physik, 417, 645-672. https://doi.org/10.1002/andp.19364170706

[19] Spinelli, P.M., Verschuuren, A. and Polman, A. (2012) Broadband Omnidirectional Antireflection Coating Based on Subwavelength Surface Mie Resonators. Nature Communications. https://doi.org/10.1038/ncomms1691 
[20] Wang, Z.Y., Zhang, R.J., Wang, S.Y., Lu, M., Chen, X., Zheng, Y.X., Chen, L.Y., Ye, Z., Wang, C.Z. and Ho, K.M. (2015) Broadband Optical Absorption by Tunable Mie Resonances in Silicon Nanocone Arrays. Nature. https://doi.org/10.1038/srep07810

[21] Bezares, F.J., Long, J.P., Glembocki, O.J., Guo, J.P., Rendell, R., Kasica, W.R., Shirey, L., Owrutsky, J.C. and Caldwell, J.D. (2013) Mie Resonance-Enhanced Light Absorption in Periodic Silicon Nanopillar Arrays. Optics Express, 21, 27587-27601. https://doi.org/10.1364/oe.21.027587 